Journal of Computer Science 6 (10): 1062-1064, 2010

ISSN 1549-3636

(C) 2010 Science Publications

\title{
Method of Fingerprint Identification
}

\author{
Surachai Panich \\ Srinakharinwirot University, 114 Sukhumvit 23, \\ Bangkok 10110, Thailand
}

\begin{abstract}
Problem statement: The main task of this study was finding out an effective algorithm in order to match two fingerprints taken from database. The fingerprint identification techniques used in a small database in order to find out an effective algorithm to develop the accuracy in matching process. Higher-level application of this proposed algorithm was determined. Approach: The main objectives in this study were the construction of fingerprint database and matching algorithm for comparison of two fingerprints. Results: In this study, Visual $\mathrm{C}^{++}$with the library software Open CV implemented the proposed algorithm. The main program operated in two distinct modes. Conclusion: Fingerprint identification was that utilized the frequency and orientation information available in a fingerprint. The technique exploited both local and global characteristics in a fingerprint image to verify an identity.
\end{abstract}

Key words: Fingerprint identification, image matching, fingerprint database

\section{INTRODUCTION}

Forensic history had clearly shown that fingerprint is unique and permanent. Finger identification is extremely reliable. Fingerprints are the most widely used and have the longest history in real world law as shown in Fig. 1. With the rapid development of technology, especially in integrated technique and computer science, fingerprints identification system is low enough in cost so that it is not only use in forensic fields but also in civil fields.

Fingerprint Identification focuses on studying fingerprint identification techniques used in a small database in order to find out an effective algorithm to develop the accuracy in matching process (Sha et al., 2003). The image correlation approach is based on global pattern matching between an enrolled fingerprint and the given fingerprint to be matched. After two images are aligned, they are checked for correspondences.

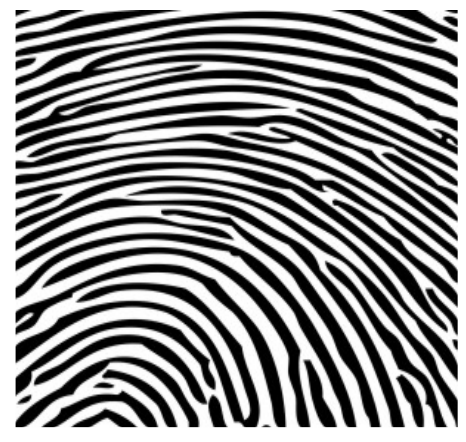

Fig. 1: Main flowchart of fingerprint
In general, this kind of computation requires less computation but is less robust against image distortion, which are unavoidable in fingerprint matching because the finger are elastic and not rigid. It is desire to obtain representations for fingerprints, which are translation and rotation invariant. Fingerprints have some extraordinary landmark structures and the combination of them could be used for specifying the main characteristics in fingerprint image (Hong et al., 1998). Reference point, which is defined as the point of maximum curvature of the concave ridge is the best choice for preserving the translation invariant. In a gray-scale fingerprint image, ridges and valleys in a local neighborhood form a sinusoidal-shaped plane wave, which has a well-defined local frequency and orientation. The sinusoidal-shaped wave varies slowly in a local constant orientation (Galbiati, 1990). Therefore, the band-pass filter that is tuned to the corresponding frequency and orientation can efficiently remove the undesired noise and preserve the true ridge and valley structure.

\section{MATERIALS AND METHODS}

In order to complete all the objectives of this study, the proposed fingerprint identification system focuses on four main tasks:

- Construction of the fingerprint images database

- Development of matching algorithm to compare fingerprint images from the database

- Test and evaluate the proposed matching algorithm 
Database collection: The experimental results in this study is conducted and verified. The fingerprint images are captured under the standard established. The database stores the fingerprint images from distinct persons (Rehan and Rashid, 2004). The collected fingerprint images are good in academic testing purpose because they were captured at different level of quality. There are fingerprint images in high gray-scale contrast, low contrast and noise. There are also images captured with invariant in location and rotation.

Data pre-processing: In general, due to the discrete nature of the variable, the histogram of the processed image is not been uniform. Histogram equalization achieves enhancement by spreading the levels of the input image over the full range of the intensity scale.

Template configuration: The required of interest region is combined from all sectors, which can be determined by step in Fig. 2.

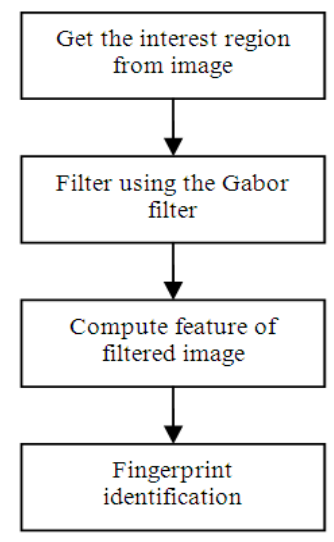

Fig. 2: Main flowchart of fingerprint

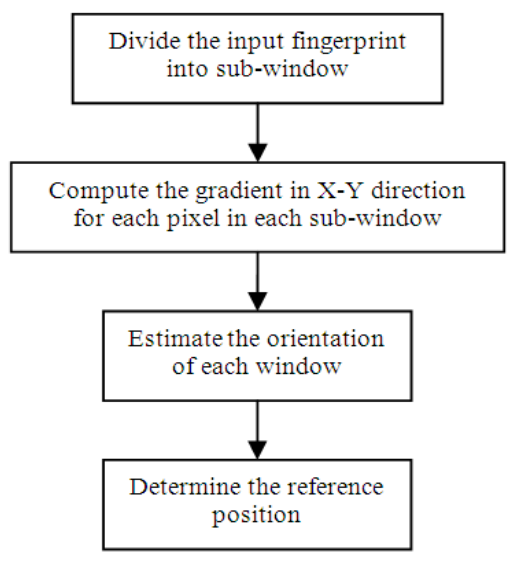

Fig. 3: Flowchart of reference point location
Reference point location: The proposed method of reference point location based on multiple resolution analysis of the orientation fields as illustrate in the flow chart in Fig. 3.

\section{RESULTS}

Software development: In this study, Visual $\mathrm{C}^{++}$with the library software Open CV use to implement the proposed algorithm. The main program operates in two distinct modes.

Database construction: The fingerprint image database is constructed by using 100 volunteers'. When active the system, user is firstly required to give his assigned user name, then, choose his own fingerprint image. If the chosen fingerprint image is his fingerprint, the system will be active the service. In this software the service is simplified to displaying the user portrait. If the chosen fingerprint does not belong to the user, the system will reject the service.

Fingerprint matching: In Fingerprint Matching algorithm Greenberg et al. (2000) the two codes of fingerprint are compared as shown in Fig. 4. After the correspondent code of fingerprint from user is used as input code, the fingerprint correspond with user will be shown on the monitor. The algorithm will compare the input of fingerprint code with the database.

If fingerprint is matched with database, the software will show the message of matched fingerprint. If it is not matched, it will display a warning.

\section{DISCUSSION}

To take the algorithm in to real application, it needs to overcome the two problems, which are the problem with time cost in getting the finger matching and the determining the local orientation. To apply the proposed program in the real life application, the key obstacle need to overcome is decreasing the time cost in getting the finger matching.

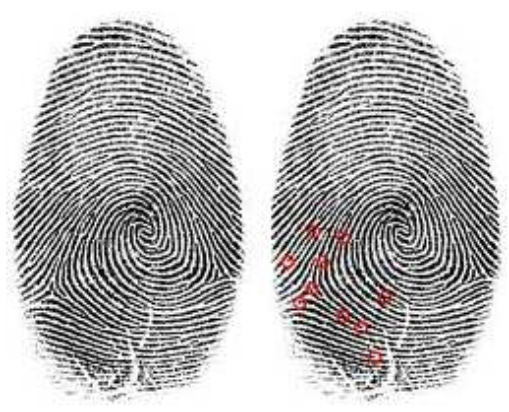

Fig. 4: Fingerprint matching of two images 


\section{CONCLUSION}

In this study, fingerprint identification is that utilized the frequency and orientation information available in a fingerprint. The technique exploited both local and global characteristics in a fingerprint image to verify an identity. The matching stage computes the Euclidean distance between the Finger Codes. The fingerprint images quality is developed by using histogram equalization and normalization before filtered by Gabor filter. The algorithm reached high accuracy when perform in small database of 100 fingerprint images. However, some problems need to be solved in the future. The main difficulty in this study is the time cost in the finger matching. The Gabor filter takes time to convolve the input fingerprint image with eight Gabor filters. Beside, it is very difficult to locate the right reference point because the limitation in determine the local orientation in the input fingerprint.

\section{ACKNOWLEDGEMENT}

This research from Measurement and Mobile Robot Laboratory (M and M-LAB) was supported by Faculty of Engineering, Srinakharinwirot University.

\section{REFERENCES}

Greenberg, S., M. Aladjem and D. Kogan, 2000. Fingerprint image enhancement using filtering techniques. Real-Time Imag., 8: 227-236. DOI: 10.1006/rtim.2001.0283

Galbiati, L., 1990. Machine Vision and Digital Image Processing Fundamentals. Facsimile Edn., Prentice-Hall, Eaglewood Cliffs, New Jersey, ISBN: 10: 013542044X, pp: 180.

Hong, L., Y. Wan and A. Jain, 1998. Fingerprint image enhancement: Algorithm and performance evaluation. IEEE Trans. Patt. Anal. Mach. Intell., 20: 777-789.

Rehan, N. and K. Rashid, 2004. Multi-matcher based fingerprint identification system. J. Applied Sci., 4: 611-618.

Sha, L., F. Zhao and X. Tang, 2003. Improved fingercode for filteband-based fingerprint matching. IEEE.

http://ai.pku.edu.cn/aiwebsite/research.files/collected\%2 0papers\%20-

$\% 20$ fingerprint/Improved\%20fingercode\%20for\%20filte rbank-based\%20fingerprint\%20matching.pdf 